\title{
Parent and reduced graphene oxide of different origin in light of neutron scattering
}

\author{
E. F. Sheka ${ }^{1}$, I. Natkaniec ${ }^{2}$, N. N. Rozhkova ${ }^{3}$, E. Yu. Buslaeva ${ }^{4}$, \\ S. V. Tkachev ${ }^{4}$, S. P. Gubin ${ }^{4}$, V.P. Mel'nikov ${ }^{5}$ \\ ${ }^{1}$ Department of Theoretical Physics and Mechanics, Peoples' Friendship \\ University of Russia, Moscow, Russia \\ ${ }^{2}$ Faculty of Physics, Adam Mickiewicz University, Poznań, Poland \\ ${ }^{3}$ Institute of Geology, Karelian Research Centre RAS, Petrozavodsk, Russia \\ ${ }^{4}$ Kurnakov Institute of General and Inorganic Chemistry, RAS, Moscow, Russia \\ ${ }^{5}$ Semenov Institute of Chemical Physics RAS, Moscow, Russia \\ sheka@icp.ac.ru, inat@amu.edu.pl
}

\section{PACS 61.05.fg, 61.46.+w, 63.22._m, 81.05.Uw DOI 10.17586/2220-8054-2016-7-1-71-80}

The current paper presents results from an extended neutron scattering study of a three-part set of parent and reduced graphene oxides ( $\mathrm{GO}$ and $\mathrm{rGO}$, respectively) of different origins. The first part concerns the rGO of natural origin represented by shungite carbon, the second and third parts are related to synthetic GO/rGO pairs with the latter produced during either chemical treatment or via thermal exfoliation of the parent GO, respectively. The study involved both the neutron diffraction and inelastic neutron scattering. The one-phonon amplitude-weighted density of vibrational states $G(\omega)$ represents the inelastic incoherent neutron scattering spectra of the products. Common characteristics and individual distinctions of the studied species are discussed.

Keywords: graphene oxide, reduced graphene oxide, shungite carbon, retained water, inelastic neutron scattering, neutron diffraction.

Received: 20 November 2015

\section{Introduction}

Graphene oxide and reduced graphene oxide have become the favorite species for neutron scattering study [1-3]. The hydrophilicity of GO and the hydrophobicity of rGO are responsible for the intense responses in both cases. The former is promoted by a considerable retaining of water between individual GO sheets as well as with hydroxyls that are formed during the interaction of the retained water with epoxy groups on the GO basal plane [3]. In the second case, the degree to which the rGO circumference is occupied with hydrogen depends on the rigour of the reduction conditions. $\mathrm{C}-\mathrm{H}$ bonds formed during the reduction actively participate in scattering via vibrational excitation of both directly bound and involved in the vibrations of the carbon core by 'riding effect'. Combined with the superb sensitivity for neutron scattering on protium, the above features provide a large hydrogen-sensitive method. In practice, the hydrogen saturation of both $\mathrm{GO}$ and $\mathrm{rGO}$ is quite variable and dependent on the chemical protocols for its production and conditions of their storage. The current paper presents the main findings related to the response of neutron scattering to high variability of $\mathrm{GO}$ and rGO products.

Three types of objects have been studied. The first is the naturally-sourced rGO represented by shungite carbon (sh-rGO below) [1,2]. The second covers synthetic GO and rGO products of the Akkolab company [5] (Ak-GO and Ak-rGO) with the latter produced in the course of chemical treatment [6]. The third presents a pair of synthetic GO/rGO products, the 
former of which is a free standing GO paper (ppGO) while the latter is produced via the thermal exfoliation of the parent ppGO (TErGO) [4]. The atom mass contents of the samples alongside with the per-one-benzenoid-ring chemical formulae are given in Table 1.

TABLE 1. Chemical composition of GO and rGO products, wt $\%$

\begin{tabular}{|c|c|c|c|c|c|}
\hline Products & $\mathrm{C}$ & $\mathrm{O}$ & $\mathrm{H}$ & $\begin{array}{c}\text { Chemical formula per } \\
\text { one benzoid ring }\end{array}$ & References \\
\hline $\begin{array}{c}\text { Shungite } \\
\text { carbon }\end{array}$ & $95.5 \pm 0.6$ & $3.3 \pm 0.4$ & $2.0-0.7$ & $\mathrm{C}_{6} \mathrm{O}_{0.1} \mathrm{H}_{1.6-0.7}$ & {$[7]$} \\
\hline Ak-rGO & $92.0 \pm 1.0$ & $5.5 \pm 0.5$ & $1.5 \pm 0.5$ & $\mathrm{C}_{6} \mathrm{O}_{0.3} \mathrm{H}_{1.2}$ & {$[6]$} \\
\hline TErGO & $87.1 \pm 0.3$ & $12.1 \pm 0.3$ & $0.5 \pm 0.3$ & $\mathrm{C}_{6} \mathrm{O}_{0.7} \mathrm{H}_{0.4}$ & {$[4]$} \\
\hline Ak-GO & $58.0 \pm 1.0$ & $39.0 \pm 1.0$ & $1.5 \pm 0.5$ & $\mathrm{C}_{6} \mathrm{O}_{3} \mathrm{H}_{2}$ & {$[6]$} \\
\hline ppGO & $56.2 \pm 0.3$ & $40.5 \pm 0.3$ & $0.7 \pm 0.3$ & $\mathrm{C}_{6} \mathrm{O}_{3} \mathrm{H}_{0.85}$ & {$[4]$} \\
\hline
\end{tabular}

The study involved both the neutron powder diffraction (NPD) and inelastic neutron scattering (INS). Both studies were performed using the inverted-geometry time-of-flight (TOF) spectrometer NERA [8] at the high flux pulsed IBR-2 reactor of the Frank Laboratory of Neutron Physics at the Joint Institute for Nuclear Research.

\section{General remarks concerning NPD study}

Figure 1 shows a typical picture of the NPD patterns related to the GO and rGO samples. The picture is common for all the studied samples in general, while differing slightly in details concerning the width and position of the rGO and GO peaks at $\sim 3.5 \AA$ and $\sim 7 \AA$ that are equivalent to the allowed $\operatorname{Gr}(002)$ and forbidden $\operatorname{Gr}(001)$ reflexes of graphite, respectively. Both peaks are broadened, which gives evidence for a stacked structure with a relevant interlayer distance. The obtained data concerning the interlayer spacing in the studied samples, as well as the lateral dimension of the layer, are given in Table 2 (details of the NPD study analysis are given elsewhere [1-4]). As seen in the table, all the studied samples present graphite-like stacked structures differing therewith by both the number of layers in the relevant stacks and the lateral dimensions of the layers. The latter was estimated by the width of Gr(110)-like peaks positioned at $\sim 1.2 \AA$.

TABLE 2. NPD structural data

\begin{tabular}{|c|c|c|c|c|}
\hline Samples & $\begin{array}{c}\text { Peak } \\
\text { position }(\AA)\end{array}$ & $\begin{array}{l}\text { Peak width } \\
\text { FWHM }(\AA)\end{array}$ & $\begin{array}{l}\text { Number } \\
\text { of layers }\end{array}$ & $\begin{array}{c}\text { Layer lateral } \\
\text { dimension }^{1}(\mathrm{~nm})\end{array}$ \\
\hline Graphite & $3.35 \pm 0.0002$ & $0.034 \pm 0.001$ & $\sim 100$ & $>20$ \\
\hline sh-rGO & $3.45 \pm 0.0015$ & $0.548 \pm 0.006$ & $5-6$ & $3-4$ \\
\hline Ak-rGO & $3.50 \pm 0.01$ & $0.30 \pm 0.01$ & 7 & $>20$ \\
\hline TErGO & $3.36 \pm 0.01$ & $0.26 \pm 0.01$ & 8 & $>20$ \\
\hline Ak-GO & $7.00 \pm 0.01$ & $1.00 \pm 0.01$ & $2-3$ & $>20$ \\
\hline ppGO & $7.21 \pm 0.01$ & $0.50 \pm 0.01$ & $4-6$ & $>20$ \\
\hline
\end{tabular}

${ }^{1}$ The definition $>20 \mathrm{~nm}$ marks the low limit of the lateral dimension of layers pointing that it is bigger than the coherent scattering region of crystalline graphite equal to $\sim 20 \mathrm{~nm}$ along $c$ direction [9]. Actual layer dimensions are of microsize range $[3,4]$. 


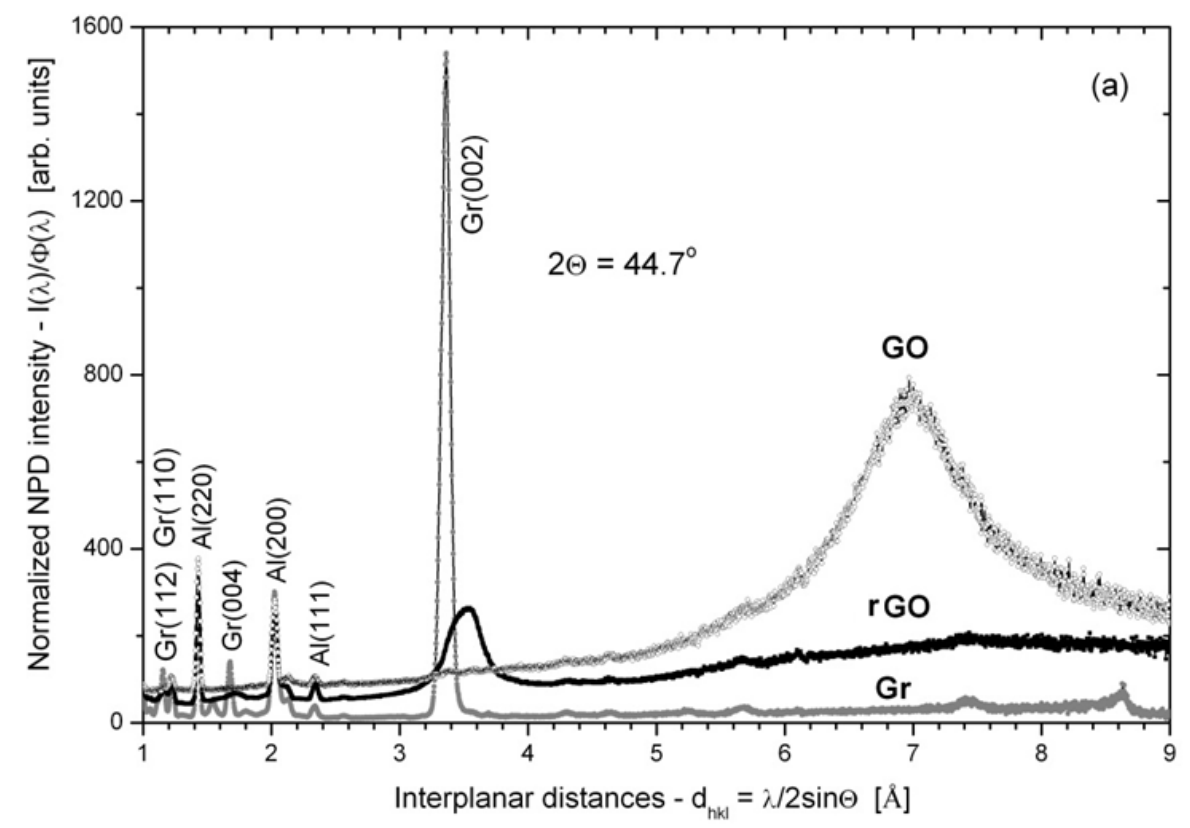

FIG. 1. NPD of spectral graphite (Gr) and Akolab products - graphene oxide (GO) and reduced graphene oxide (rGO), $T=20 \mathrm{~K}$ [3]. Gr(hkl) (black) and $\mathrm{Al}(\mathrm{hkl})$ (gray) denote characteristic diffraction peaks of spectral graphite and cryostat (alongside with sample holder) aluminum at different Miller indexes, respectively

\section{General characteristics of the INS study}

Typical INS TOF spectra for synthetic GO and rGO versus the incident neutrons' wavelength at low and room temperatures are shown in Fig. 2; the results were exempted from the background and carbon atoms scattering and identically normalized by the monitor counter. The temperature dependence is typical for molecular solids and is provided by considerable enhancement of multi-phonon scattering and a significant smoothing of the entire spectrum as a whole [10-12]. Both species scatter the neutrons quite intensely, indicating to be hydrogenenriched. However, the hydrogenation is quite different in these two cases. While the hydrogen atoms in retained water play the main role in the hydrophilic GO, whose spectrum is definitely akin to that of wet graphite oxide (GrO) [13], the ones incorporated in the carbonaceous core of hydrophobic rGO are responsible in the second case. Synthetic samples were intensely dried before measuring. As for shungite carbon, the pristine sample contains a considerable amount of retained water $(\sim 4 \mathrm{wt} \%)$ [2] which can be removed by prolonged heating at $100{ }^{\circ} \mathrm{C}$ under soft vacuum. The transformation of the TOF INS spectrum when passing from wet (1) to dried (2) shungite is well seen in Fig. 3. The difference between the spectra is due to released water.

Experimentally, differential cross-section is the measure of the INS intensity. It consists of two parts:

$$
\frac{d^{2} \sigma}{d \Omega d E}=\left(\frac{d^{2} \sigma}{d \Omega d E}\right)_{c o h}+\left(\frac{d^{2} \sigma}{d \Omega d E}\right)_{i n c}=\sum_{n} \frac{\left(b_{n}^{c o h}\right)^{2}}{M_{n}} S_{n}^{c o h}(Q, \omega)+\sum_{n} \frac{\left(b_{n}^{i n c}\right)^{2}}{M_{n}} S_{n}^{i n c}(Q, \omega),
$$

that present coherent and incoherent INS [14]. Lengths of neutron scattering, $b_{n}^{\text {coh }}$ and $b_{n}^{\text {inc }}$, as well as mass of the $n$-th nucleus $M_{n}$, different for atoms and isotopes of the same atoms, determine the contribution of a particular nucleus towards the total INS intensity. The presence 


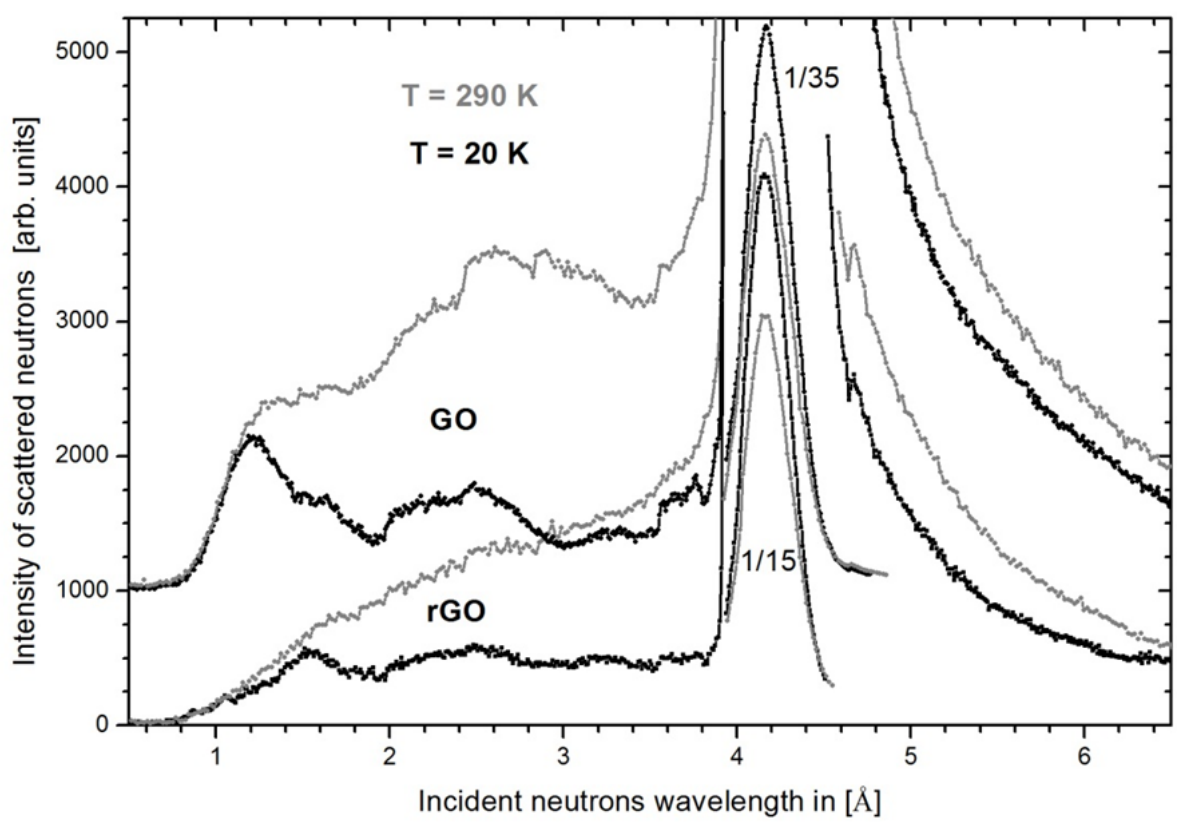

FIG. 2. Time-of-flight INS spectra of Ak-GO and Ak-rGO, recorded at $T=20 \mathrm{~K}$ (black) and $295 \mathrm{~K}$ (light gray) normalized by monitor counter to the same number of incident thermal neutrons flux for the wavelength region $(0.8-6.8) \AA$, and exempted of background and scattering spectrum of graphite. The intensity of elastic peaks of Ak-GO and Ak-rGO spectra is 35- and 15-fold reduced, respectively [3]

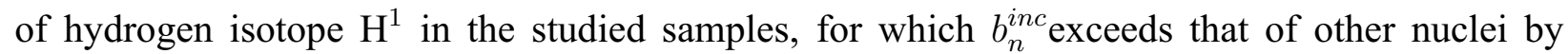
several times, permits us to use the incoherent inelastic neutron scattering (IINS) cross-section to calculate the INS spectral intensity. In the framework of commonly used inelastic incoherent one-phonon scattering approximation, this is expressed as [14]:

$$
\sigma_{1}^{i n c}\left(E_{i}, E_{f}, \varphi, T\right) \approx \sqrt{\frac{E_{f}}{E_{i}}} \frac{\hbar\left|Q\left(E_{i}, E_{f}, \varphi\right)\right|^{2}}{\omega} \sum_{n} \frac{\left(b_{n}^{i n c}\right)^{2}}{M_{n}} \frac{\exp \left(-2 W_{n}\right)}{1-\exp \left(-\frac{\hbar \omega}{k_{B} T}\right)} G_{n}(\omega),
$$

where, $Q\left(E_{i}, E_{f}, \varphi\right)$ is the neutron momentum transfer; $\omega=\left(E_{i}-E_{f}\right)$ is the neutron energy transfer; $\exp \left(-2 W_{n}\right)$ is the Debye-Waller factor; $G_{n}(\omega)$ presents the $n$-th atom amplitudeweighted density of vibrational states (AWDVS):

$$
G_{n}(\omega)=\sum_{j}\left[A_{j}^{n}(\omega)\right]^{2} \delta\left(\omega-\omega_{j}\right)
$$

Here, $A_{j}^{n}(\omega)$ is the $n$-th atom contribution into the eigenvector of the $j$-th phonon mode expressed as the amplitude of the $n$-th atom displacement at the vibrational frequency $\omega_{j}$.

If the matter consists of different nuclei, each nuclear family superpositionally enters the total scattering intensity. The maximum contributions of $\mathrm{H}^{1}, \mathrm{C}^{12}$, and $\mathrm{O}^{16}$ isotopes form a series $639: 3.7: 2.1$, thus showing that under equal conditions, the scattering from protium atoms are 173 and 304 times stronger than that of carbon and oxygen atoms, respectively. This allows us to neglect the contribution of both heavy atomic families into the total scattering intensity thus presenting it as a mononuclear quantity : 


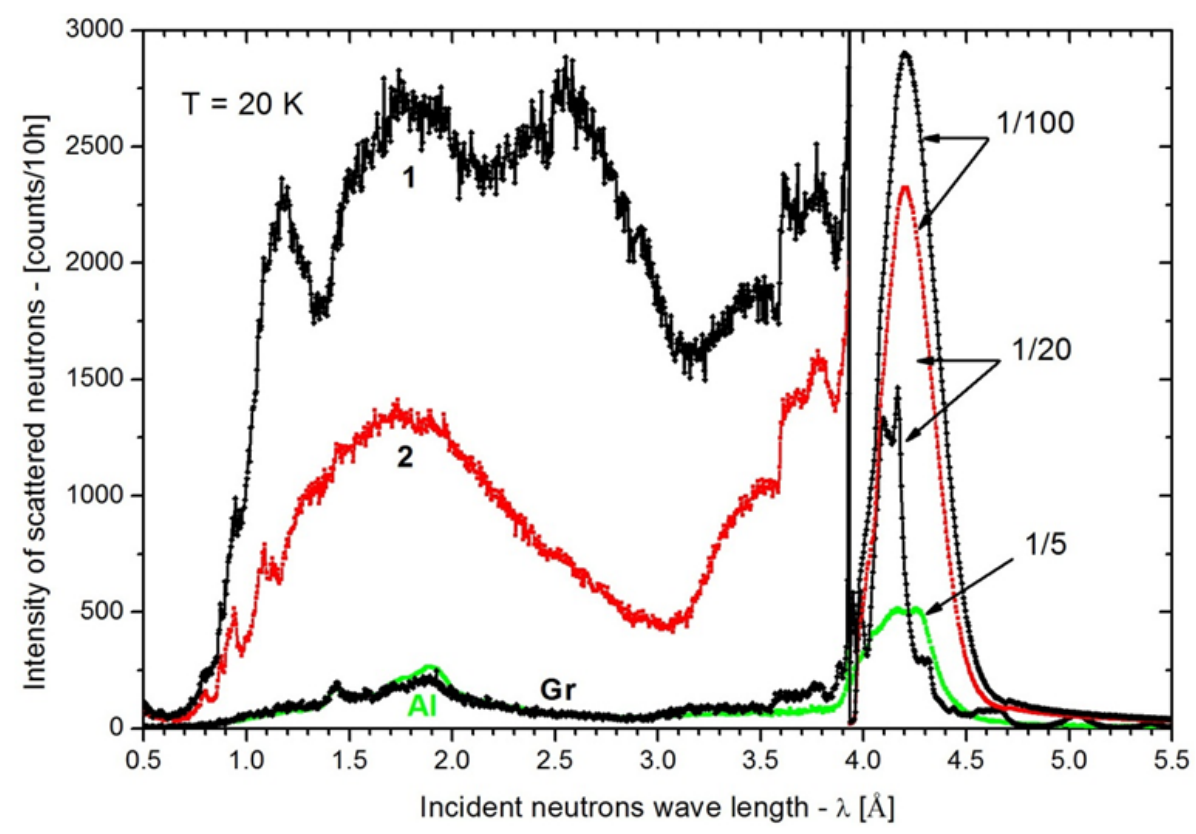

FIG. 3. Time-of-flight INS spectra from wet (1) and dried (2) shungites and spectral graphite (Gr) [1]. Curve Al presents background from Al cryostat and sample holder material. The intensity of elastic peaks is 100 -fold, 20 -fold and 5-fold reduced for spectra 1, 2, Gr, and background, respectively. Spectra are summarized over 15 scattering angles and normalized per 10 hours exposition time at constant power of the IBR-2 equal 1.9 MW

$$
G(\omega)=\sum_{n} G_{n}(\omega)
$$

which is the hydrogen-partitioned AWDVS. A negligible contribution of carbons atoms in the studied IINS spectra, which cannot be distinguished from background, is amply demonstrated in Fig. 3.

\section{Comparative analysis of the experimental $G(\omega)$ spectra}

\subsection{Retained water spectra}

The IINS $G(\omega)$ spectra of the studied samples form two distinct groups. The first is related to GO partners of the synthetic families as well as to the retained water in shungite carbon while the second covers spectra of dried shungite (sh-rGO) and two other synthetic rGOs. Spectra of the first group are given in Fig. 4. The water of shungite carbon (sh-water), presented in Fig. $4 \mathrm{~b}$, is the $G(\omega)$ image of the difference between spectra 1 (wet shungite) and 2 (dried shungite) in Fig. 3. Additionally, the NERA spectrum for Ih ice is given in Fig. 4a for comparison. Since the relevant GO matrices of Ak-GO and ppGO are not hydrogen-rich and consist of carbon and oxygen atoms [3,4], their IINS spectra should be attributed primarily to confined water, whose $G(\omega)$ spectra are presented in Fig. 4c and d.

As seen in the figure, evidently, the water spectra of the studied samples have much in common: all of them involve three parts characteristic for the bulk water, namely: intensitydominant $\mathrm{B}$ band in the $500-600 \mathrm{~cm}^{-1}$ region, less intense $A$ and $L$ bands in the $\sim 100$ and $200-300 \mathrm{~cm}^{-1}$ regions. These peculiarities are undoubted reflections of similar features for bulk water (ice) spectrum and can be attributed to rotational (libration) modes (B) and hindered 

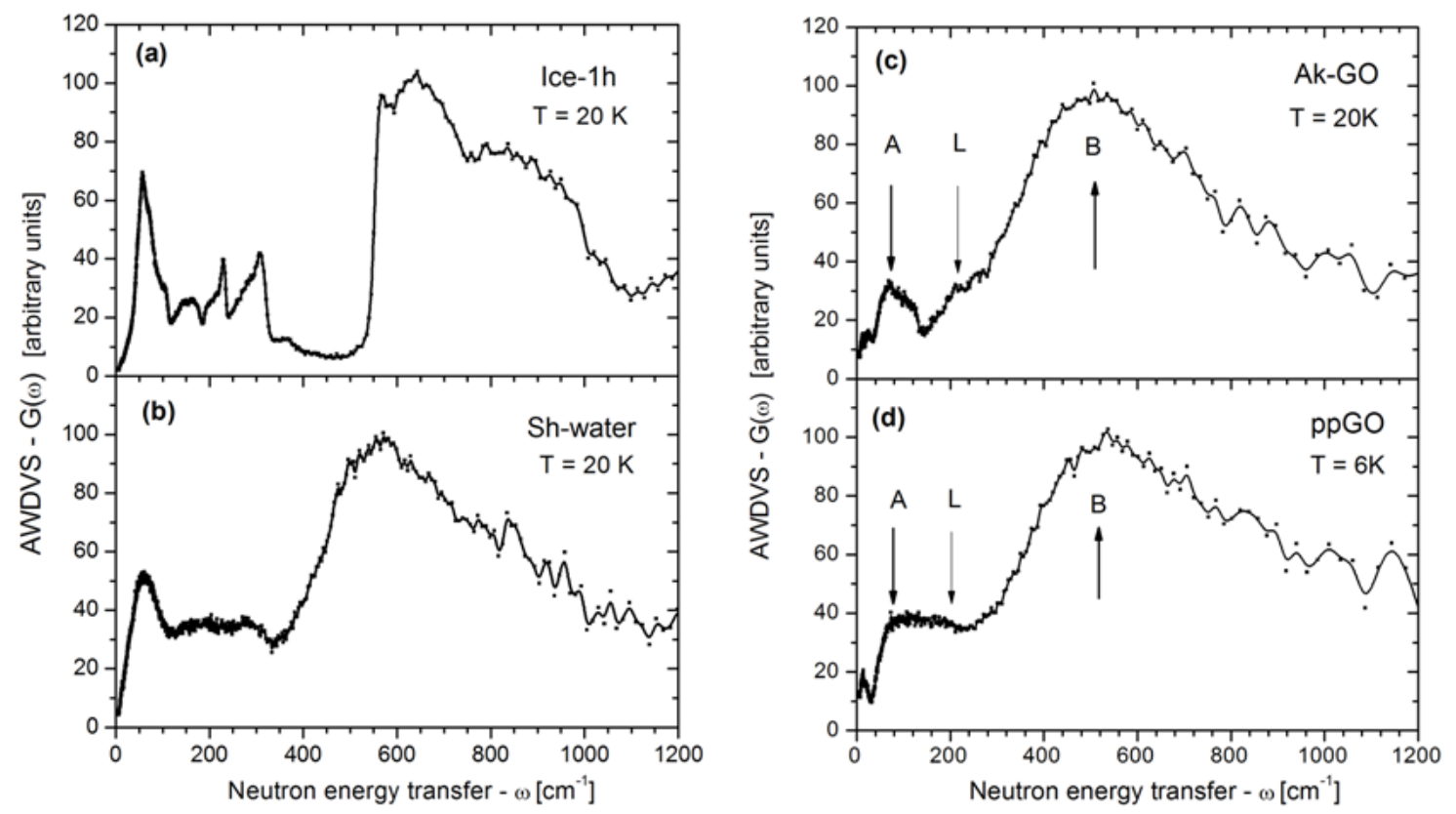

FIG. 4. Experimental amplitude-weighted density of vibrational states (AWDVS) $G(\omega)$ spectra: bulk water ( $I_{h}$ ice) (a) and retained water in shungite (b), AkGO (c) and ppGO (d)

translational mode $(A$ and $L)$. As it is well known [15], both the hindered translational and rotational modes are present in bulk water because of intermolecular hydrogen bonds (HB) that are formed by each water molecule surrounded by four other ones. Evidently, the configuration changes when water molecules cannot move freely because of confining geometry due to which it is quite natural to expect a visible modification of water's spectrum when passing from bulk to retained water. As shown in Fig. 4, the spectrum of retained water differs remarkably from that of the bulk compound. However, the less change is characteristic for sh-water spectrum while in the case of the Ak-GO and ppGO spectra of GO samples, the changes are so considerable that one can speak about a drastic modification of the water molecule's behavior (see detailed discussion in [3]); therewith, both latter spectra are quite similar. The observed peculiarities are a result of the confined geometries in which water is bound.

The INS study of retained water has a long history, over the course of which, among other important issues, considerable attention has been given to the topology of its confined geometries [16-20]. According to suggested classification, the sh-water spectrum should be attributed to the spectrum of water retained in pores while Ak-GO and ppGO spectra are characteristic for interlayer confinement. This conclusion is well consistent with our presentation for the sample structure. In reality, the detailed adsorption [21] and small-angle neutron scattering (SANS) [22] studies have shown that shungite carbon is a peculiar porous material with two sets of pores of $<10 \mathrm{~nm}$ and $\sim 100 \mathrm{~nm}$ lateral dimensions formed by stacks (of $4-7$ layers according to Table 2) of hydrophobic nanosize rGO sheets [23]. Conversely, laterally extended 2 - 4 layer stacks of hydrophilic GO willingly retain water between the layers in a manner similar to GrO [13].

While, as shown [13], the retained water spectrum of GrO remarkably depends on the hydration degree, a general view on $G(\omega)$ spectrum of water retained in a porous matrix [16, $18,24,25]$ does not reveal such an effect. This means that the water interaction with the inner surface of the pores is weak and the molecules can move quite freely both near the surface and 
in the pore depth. The latter is supported by the sh-water spectrum which deviates negligibly from the bulk water spectrum (see Fig. 4b). Nevertheless, in spite of weak interaction, the pore topology causes the spectrum deviations that can be observed. They concern flattering and downshift of bulk HB bends at $\sim 150 \mathrm{~cm}^{-1}$ and HB stretches at $\sim 224$ and $\sim 296 \mathrm{~cm}^{-1}$. A certain modification of bulk spectrum takes place in the region of librational modes forming a broad band in the $600-1200 \mathrm{~cm}^{-1}$ region. The latter is provided with water molecule rotations around three symmetry axes whose partial contribution determines the band shape [16]. The modes conserve their dominant role in the spectra of retained water, despite being downshifted. Obviously, the spectrum three-ax partial contribution is sensitive to the pore size [16]. Thus, the downshift of the red edge of band B from $550 \mathrm{~cm}^{-1}$ to $320 \mathrm{~cm}^{-1}$ when going from the bulk water to retained one in shungite carbon highlights the shungite pore size of a few nm, which is very consistent with the adsorption and SANS data [21,22].

In contrast with the sh-water spectrum, the Ak-GO and ppGO spectra in Figs. 4c and d considerably deviate in the region of translational modes (covering both HB bending $(A)$ and stretchings $(L)$ ) while the intense librational band $B$ is broadened and downshifted in a similar fashion to the $B$ band in the sh-water spectrum. As was shown in [3], the effect is caused by the reconstruction of the HB surrounding caused by the interaction with the GO substrates. Evidently, the effect depends on the chemical composition of the GO basal planes, the number of GO layers in stacks, the shape of the relevant GO sheets, the lateral size of the sheets, and other factors. Due to high variability of GO materials with regards to these parameters [7,26-30], one should expect widely variable INS spectra for samples, which was observed in the two studied cases.

\section{2. rGO spectra}

By analyzing IINS $G(\omega)$ spectra of rGO samples, one might conclude that a significant portion of the IINS intensity is caused by the hydrogens present on the carbonaceous skeletons. The fixation of the interlayer distance in the rGO stacks in all the studied cases from $3.4-$ $3.6 \AA$ proves that the relevant hydrogen atoms occupy the circumference $(c c f)$ area of the rGO sheets, and as a result, the latter should be attributed to framed $c c f$ oxyhydrides of graphene (FOHG). Similar to GOs, FOHGs are highly variable by chemical composition, size, and shape $[2,7,26-30]$ and unsurprisingly, their properties also widely change. The latter should be naturally attributed to the IINS spectra as well. However, since the hydrogen contribution mainly provides the IINS spectral data, one should expect some similarity in the spectral behaviors. This turns out to be true, which is illustrated by Fig. 5 .

Curves in the left $(\mathrm{a}-\mathrm{c})$ panels of Fig. 5 present the $G(\omega)$ spectra for the studied samples recorded with the NERA spectrometer at IBR-2 at JINR (NERA spectra below). The spectra clearly demonstrate a general similarity for the rGO bodies while their difference in details can be considered as convincing proof of the matter variability. Not being satisfied with these general statements, we decided to further scrutinize these results in greater detail. The obtained spectra are provided by the motion of two groups of atoms, namely: hydrogens located in the $c c f$ regions and carbons of the graphene-like cores. Vibrational frequencies follow this division and allow attribution of the spectral region below $800 \mathrm{~cm}^{-1}$ to graphite-like modes, and above that region - to vibrations of $\mathrm{C}-\mathrm{H}$ bonds mainly $[3,32]$. The fact that the low-frequency vibrational modes are well pronounced in the discussed spectra is associated with the 'riding effect' caused by the participation of hydrogen atoms displacements in the wave vectors of the carbon atom modes (see detailed discussion of the effect in [3]). In light of this, the difference in the (a) - (c) spectra may result from two facts related to changes in either the chemical composition of the $c c f$ framing of the studied FOHG or in the graphite-like vibrational modes 

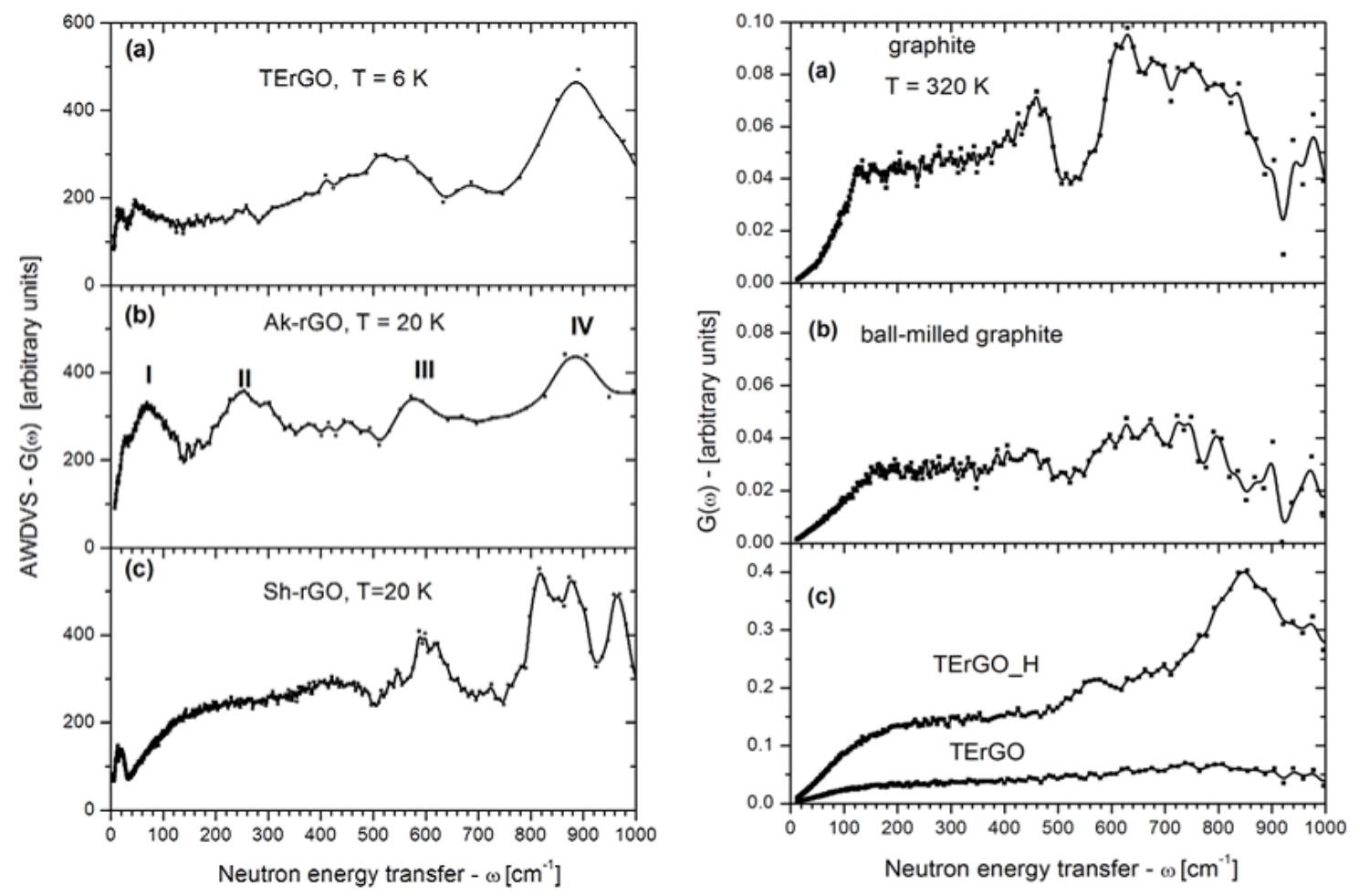

FIG. 5. Experimental amplitude-weighted density of vibrational states (AWDVS) $G(\omega)$ spectra. Left - NERA spectra: (a) TErGO [4]; (b) Ak-rGO [3]; and (c) shrGO [1,2]. Right - ILL spectra: (a) graphite; (b) bill-milled nanographite; (c) thermally exfoliated rGO (TEGO) and hydrogenated TEGO (H-TEGO) recorded at the IN4 spectrometer at the HFR in the ILL [31]

of the relevant carbon cores. Such assessment of the situation has recently obtained serious support based on the study of a set of graphite-like and graphene-like samples at the thermal neutron source of the Institute of Laue and Langevin (ILL) in Grenoble [31].

Thanks to kind permission of the authors (S.R. and C.C.), $G(\omega)$ spectra in the right (a c) panels of Fig. 5 reproduce data obtained in the ILL (ILL's spectra below). In contrast to the NERA experimental facilities, the latter related to those at the ILL HFR allowed to fix the coherent INS from carbon atoms, providing parent (a), ball-milled (b) graphite and graphene TEGO (c) scattering to be above the background. The latter sample presents rGO obtained via the thermal exfoliation of GrO (produced from the parent graphite as well) under an inert atmosphere. Taken together, the ILL carbon data exhibit changes in the $G(\omega)$ spectra when going from macroscopic graphite to mesoscopic ball-milled graphite and nanoscopic TEGO, thus disclosing changes in the behavior of graphite-like phonon modes due to decreasing size parameters [31]. Clearly, while $G(\omega)$ spectrum of graphite (a) significantly reproduces all the features of the calculated density of states (DOS) of graphite [33] the reduced dimensions of the grains in bill-milled graphite (b) and TEGO (c) have strong effects on the dynamic properties: peaks in the density of states progressively broaden, with an increased proportion in the low frequency part of the spectrum [31].

When comparing the discussed ILL $G(\omega)$ spectra with the NERA ones present in the left panels of Fig. 5, it is easy to note that the size reduction clearly comes out in the latter when passing from TErGO to sh-rGO within the family. Comparing the spectra of the two sets, it is clearly seen that the TErGO left spectrum is extremely similar to the right spectrum of the 
parent graphite while the right TEGO and TEGO-H spectra are very similar to the sh-rGO one. These findings are consistent with microsize lateral dimension of both TErGO and graphite samples while nanosize dimensions of sh-rGO as well as TEGO and TEGO-H are matched quite perfectly, thus making a convincing case for the decisive role of rGO sheet size for the appearance of the DOS of their vibrational states. As for the Ak-rGO spectrum, its changing from that of the TErGO and sh-rGO ones definitely proves the changing dimensions for the sample from the two mentioned above. However, the change is much more significant than caused by bill-milling, while revealing therein a particular change in the DOS spectrum for the carbons' vibrational states.

Considering both the NERA and ILL spectra, until now, we have mainly concentrated on the spectral range below $800 \mathrm{~cm}^{-1}$. However, all the NERA spectra as well as the ILL HTEGO spectrum (right (c) panel) show well-pronounced features above that region, quite similar to all the other spectra. As is shown in $[3,32]$, the region is characteristic for the non-planar deformational vibrations of $\mathrm{C}-\mathrm{H}$ units that mainly contribute into framing of FOHG under the presence of hydrogen. The observed transformation of the ILL TEGO and H-TEGO spectra, caused by the additional hydrogenation of TEGO, supports this conclusion directly.

\section{Conclusion}

The current paper presents results from an extended neutron scattering study of a set of graphene oxide $(\mathrm{GO})$ and reduced graphene oxide $(\mathrm{rGO})$ products of different origins. The neutron diffraction patterns confirmed that the stacking structures of all the species consisted of a number of layers of nanosize (natural product) and microsize (synthetic products) lateral dimension and the interlayer distances of 7.0 - 7.2 and 3.4-3.5 $\AA$ for GO and rGO, respectively. The performed INS study has convincingly shown that neutron scattering clearly distinguishes GO and rGO species and amply exhibits both common features and differences related to the compounds of both families. If the retained water in GOs and graphene-hydride nature of rGOs provide the commonality for dynamic properties within each compound family, the difference in the relevant sheet topology is responsible for a noticeable variability between them. This study has convincingly shown the topochemical nature of large polyvariance of both GO and rGO products.

\section{Acknowledgements}

The authors are grateful to S. Rols and C. Cavallari for supplying with the data obtained in the course of experiments at the IN4 spectrometer at the ILL HFR as well as for fruitful discussions and kind permition to use the data at our discretion.

\section{References}

[1] Sheka E.F., Rozhkova N.N., Natkaniec K.I., Holderna-Natkaniec K. Neutron scattering study of reduced graphene oxide of natural origin. JETP Lett., 2014, 99, P. 650-655.

[2] Sheka E.F., Rozhkova N.N., Holderna-Natkaniec K., Natkaniec I. Nanoscale reduced-graphene-oxide origin of shungite in light of neutron scattering. Nanosystems: Phys. Chem. Math., 2014, 5 (5), P. 659-676.

[3] Natkaniec I., Sheka E.F., et al. Computationally supported neutron scattering study of parent and chemically reduced graphene oxide. J. Phys. Chem. C, 2015, 119 (32), P. 18650-18662.

[4] Sheka E.F., Natkaniec I., Mel'nikov V.P., Druzbicki K. Nanosystems: Phys. Chem. Math., 2015,6 (3), P. 378-393.

[5] AkKoLab LLC, URL: www. akkolab.ru.

[6] Tkachev S.V., Buslayeva E.Yu., et al. Reduced graphene oxide. Inorg. Mater., 2012, 48, P. 796-802.

[7] Sheka E.F., Golubev E.A. About technical graphene - reduced oxide graphene- and shungite - its natural analogue. Zn. Tehn. Fiz., 2016, 86 (submitted). 
[8] Natkaniec I., Chudoba D., et al. Parameters of the NERA spectrometer for cold and thermal moderators of the IBR-2 pulsed reactor. J.Phys.: Conf. Series, 2014, 554, 012002.

[9] Opalev S.V., Belenkov E.A. Experimental study of changing graphite structure under milling. (in Russ.) Izvestiya Chelyabinskogo Nauchnogo Centra, 2004, 3, P. 27-30.

[10] Bokhenkov E.L., Natkaniec I., Sheka E.F. Determination of density of phonon states in a naphthalene crystal on basis of inelastic incoherent neutron scattering. Zh. Exp. Teor. Fiz., 1976, 70, P. 1027-1043.

[11] Kolesnikov A.I., Bokhenkov E.L., Sheka E.F. Multiphonon coherent scattering of neutrons in a naphthalene crystal. JETP, 1984, 57, P. 1273-1278.

[12] Johnson M.R., Parlinski K., Natkaniec I., Hudson S. Ab initio calculations and INS measurements of phonons and molecular vibrations in a model peptide compound - urea. Chem. Phys., 2003, 291, P. 53-60.

[13] Buchsteiner A., Lerf A., Pieper J. Water dynamics in graphite oxide investigated with neutron scattering. $J$. Phys. Chem. B, 2006, 110, P. 22328-22338

[14] Marshall W., Lovesey S.W. Theory of Thermal Neutron Scattering, Oxford, 1971.

[15] Finney J.L. Water? What's so special about it? Phil. Trans. R. Soc. Lond. B, 2004, 359, P. 1145-1165.

[16] Crupi V., Majolino D., Migliardo P., Venuti V. Neutron scattering study and dynamic properties of hydrogenbonded liquids in mesoscopic confinement. 1. The water case. J. Phys. Chem. B, 2002, 106, P. 10884-10894.

[17] Kolesnikov A.I., Zanotti J.-M., Loong C.-K., Thiyagarajan P. Anomalously soft dynamics of water in a nanotube: a revelation of nanoscale confinement. Phys. Rev. Lett., 2004, 93, 035503.

[18] Corsaro C., Crupi, V., et al. Inelastic neutron scattering study of water in hydrated lta-type zeolites. J. Phys. Chem. A, 2006, 110, P. 1190-1195.

[19] Chen S.-H., Loong C.-K. Neutron scattering investigations of proton dynamics of water and hydroxyl species in confined geometries. Nucl. Eng. Techn., 2006, 38, P. 201-224.

[20] . Bertrand C.E., Zhang Y., Chen S.-H. Deeply-cooled water under strong confinement: neutron scattering investigations and the liquid-liquid critical point hypothesis. Phys. Chem. Chem. Phys., 2013, 15, P. 721-745.

[21] Rozhkova N.N., Emel'yanova G.I., et al. From stable aqueous dispersion of carbon nanoparticles to the clusters of metastable Shungite carbon. Glass Phys. Chem., 2011, 37, P. 613-618.

[22] Avdeev M.V., Tropin T.V., et al. Pore structures in shungites as revealed by small-angle neutron scattering. Carbon, 2006, 44, P. 954-961.

[23] Sheka E.F., Rozhkova N.N. Shungite as the natural pantry of nanoscale reduced graphene oxide. Int. J. Smart Nano Mater, 2014, 5, P. 1-16.

[24] Hall P.G., Pidduck A., Wright C.J. Inelastic neutron scattering by water adsorbed on silica. J. Colloid. Interface Sci., 1981, 79, P. 339-349.

[25] Sheka E.F., Khavryutchenko V.D., Markichev I.V. Techonological polymorphism of disperse amorphous silicas: inelastic neutron scattering and computer modelling. Russ. Chem. Rev., 1995, 64, P. 389-414.

[26] Kuila T., Bose S., et al. Chemical functionalization of graphene and its applications. Progr. Mater. Sci., 2012, 57, P. 1061-1105.

[27] Luo J., Kim J., Huang J. Material processing of chemically modified graphene: Some challenges and solutions. J. Acc. Chem. Res., 2013, 46, P. 2225-2234.

[28] Chua C.K., Pumera M. Chemical reduction of graphene oxide: a synthetic chemistry viewpoint. Chem. Soc. Rev., 2014, 43, P. 291-312.

[29] Zhao J., Liu L., Li F. Graphene Oxide: Physics and Applications. Springer, 2015.

[30] Kumar P.V., Bardhan N.M., et al. Scalable enhancement of graphene oxide properties by thermally driven phase transformation. Nature Chem., 2014, 6, P. 151-158.

[31] Cavallari C., Pontiroli D., et al. Hydrogen on graphene investigated by inelastic neutron scattering. J. Phys. Conf. Series, 2014, 554, 012009.

[32] Druzbicki K., Natkaniec I. Vibrational properties of water retained in graphene oxide. Chem. Phys. Lett., 2014, 600, P. 106-111.

[33] Bousige C., Rols S., et al. Lattice dynamics of a rotor-stator molecular crystal: fullerene-cubane $\mathrm{C}_{60} \cdot \mathrm{C}_{8} \mathrm{H}_{8}$. Phys. Rev. B, 2010, 82, 195413. 